\title{
Grape polyphenols supplementation reduces muscle atrophy in a mouse model of chronic inflammation
}

\author{
Karen Lambert Ph.D. ${ }^{a}$, Marjorie Coisy-Quivy Ph.D. ${ }^{a}$, Catherine Bisbal Ph.D. ${ }^{\text {a }}$, \\ Pascal Sirvent Ph.D. ${ }^{a}$, Gerald Hugon ${ }^{a}$, Jacques Mercier Ph.D., M.D. ${ }^{\mathrm{a}, \mathrm{b}}$, \\ Antoine Avignon Ph.D., M.D. ${ }^{\mathrm{a}, \mathrm{b}}$, Ariane Sultan ${ }^{\mathrm{a}, \mathrm{b}, *}$ \\ ${ }^{a}$ UMR CNRS 9214 - Inserm U1046 - PHYMEDEXP - Université de Montpellier, Montpellier, France \\ ${ }^{\mathrm{b}}$ CHRU Montpellier, Montpellier, France
}

Keywords:

Muscle atrophy

Polyphenols

Oxidative stress

TGF

Mitochondria

Caspases

\begin{abstract}
A B S T R A C T
Objectives: Polyphenols (PP) have demonstrated beneficial effects on low-grade inflammation and oxidative stress; however, little is known about their effect on highly inflamed muscle. The purposes of this study were (i) to evaluate muscle alteration induced by high-grade inflammation, and (ii) to test the effects of red grape PP supplementation on these alterations.

Methods: We used a transgenic mice model (transforming growth factor [TGF] mice) to develop a high T cell-dependent inflammation and C57 BL/6 control (CTL) mice model. Skeletal muscles of TGF and CTL mice were investigated for inflammation, atrophy and oxidative stress markers. Isolated mitochondria from hindlimb muscles were used for respiration with pyruvate as substrate and oxidative damages were measured by Western blot. TGF mice were supplemented with a mixture of red grape polyphenols ( $50 \mathrm{mg} / \mathrm{kg} / \mathrm{d}$ ) for $4 \mathrm{wk}$. Data were analyzed by one-way analysis of variance (ANOVA) and post hoc Bonferroni's multiple comparison tests.

Results: TGF mice presented skeletal muscle inflammation, oxidative stress, mitochondrial alteration and muscle atrophy. Atrophy was associated with two distinct pathways: (i) one linked to inflammation, NF- $\kappa$ B activation and increased ubiquitin ligase expression, and (ii) one dependent on reactive oxygen species (ROS) production leading to damaged mitochondria accumulation and activation of caspase-9 and 3. Supplementation of TGF mice with a mixture of red grape polyphenols (50 mg/kg/d) for $4 \mathrm{wk}$ improved mitochondrial function and highly decreased caspases activation, which allowed muscle atrophy mitigation.

Conclusions: These observations suggest that nutritional dosages of red grape polyphenols might be beneficial for reducing skeletal muscle atrophy, even in a high-grade inflammation environment.
\end{abstract}

\section{Introduction}

Muscle atrophy is frequently associated with a highinflammatory state such as cancer cachexia, rheumatoid arthritis, infection or chronic obstructive pulmonary disease [1,2]. Moreover, muscle atrophy is an independent predictor of increased mortality in such disorders [3,4].

Mechanisms implicated in muscle atrophy involved two proteolysis pathways: the ubiquitin-proteasome system (UPS) and the autophagy-lysosome pathway. The UPS is an essential regulator of sarcomeric protein degradation via their

Authors K. L. and M. C.-Q. contributed equally to this work.

* Corresponding author. Tel.: +33 4673384 02; fax: +33 467415231 .

E-mail address: a-sultan@chu-montpellier.fr (A. Sultan). attachment to multiple ubiquitin molecules and then their degradation by the $26 \mathrm{~S}$ proteasome. In muscle, the ubiquitination of proteins is regulated by the muscle specific ubiquitin ligase E3: muscle atrophy F-box (MAFbx)/Atrogin-1 and muscle RING-finger protein-1 (MuRF1) [5,6]. The autophagy-lysosomal pathway triggers the removal of specific organelles such as mitochondria or protein aggregates ensuring cell metabolism and cell component turnover [7,8]. This pathway begins with the capture of substrates into an autophagosome that fuses with lysosome to form an autophagolysosome. The sequestered material is subsequently degraded by lysosomal hydrolases [7]. However, during muscle proteolysis, myofilaments are first released via proteolytic action of calpain and/or caspase-3 before their degradation by proteasome, as this latter can degrade only monomeric proteins [9]. 
Activation of inflammation and oxidative stress are both involved in muscle atrophy $[10,11]$. Nuclear factor $\kappa B(N F-\kappa B)$ pathway activation leads to inflammatory cytokine production as tumor necrosis factor $\alpha$ (TNF $\alpha$ ), interleukin 6 (IL6) and interferon $\gamma$ (IFN $\gamma$ ), which plays an essential role in muscle atrophy and could activate NF- $\kappa \mathrm{B}[11]$.

Oxidative stress also contributes to muscular atrophy, through activation of the ubiquitin-proteasome pathway [12,13] and of caspase- 3 via caspase-9 [14]. Caspase- 3 seems to be the initial step triggering muscle proteolysis [15]. Inhibiting caspase-3 activation results in muscle atrophy attenuation [16].

Currently, there is no pharmacologic treatment effective in restoring or maintaining skeletal muscle (SM) mass during chronic inflammatory disease. Therefore, non pharmacological alternative care strategies need to be developed to allow improving quality of life and life expectancy in inflammatory disease as previously demonstrated with physical activity [17].

Recently, several studies determined that supplementation with polyphenols (PPs) could modulate inflammation and oxidative stress [18-20]. PPs are phytochemical compounds abundantly present in fruits and vegetables [21]. They possess aromatic ring(s) with one or more hydroxyl moieties allowing ROS scavenging [22]. Moreover, in vivo, their antioxidative capacity could also be due to their ability to regulate antioxidative enzyme expression at posttranscriptional mRNA level via ARE- and Nrf2-mediated mRNA decay [23]. PPs have also antiinflammatory capacity in that they inhibit NF- $\kappa$ B signaling [24] and expression of inflammatory genes [25]. Because of this, PP supplementation has been extensively tested in pathologies characterized by low-grade inflammation and oxidative stress, such as insulin resistance [26,27], type 2 diabetes [28] and cardiovascular diseases [29]. There is little data concerning PP supplementation effects on muscle atrophy in pathologies characterized by high-grade inflammation [30,31].

Therefore, the purposes of this study were first to evaluate muscle alteration induced by high-grade inflammation in a transgenic mice model (transforming growth factor [TGF] mice), and second, to test the effects of PP supplementation on these alterations.

\section{Materials and methods}

Ethical approval

All procedures conformed to the Directive 2010/63/EU of the European Parliament and the Council of 22 September 2010 on the protection of animals used for scientific purposes (agreement number: A34-172-38).

\section{Animal experiments}

The C57 BL/6 strain of mice used during our study was bred in our facility. The CD4 dnTGF $\beta$ RII transgenic mice or TGF mice generated by Dr Richard Flavell is deposited at Jackson Laboratory (B6.Cg-Tg (Cd4-TGFBR2)16 Flv/J, stock no 005551). This mouse model express a dominant-negative form of the TGF- $\beta$ receptor type II under the control of the murine CD4 promoter leading to the loss of T cell activation inhibition by TGF- $\beta$. These mice develop a high T cell-dependent inflammation [32,33], inducing a large systemic, liver, kidney, colon and lung inflammation $[32,33]$.

Three-month-old male CD4 dnTGFßRII transgenic (TGF, $\mathrm{n}=14$ ) and C57 BL/ B6 control ( $C T L ; n=10)$ mice were housed in plastic cages in a temperaturecontrolled environment with a 12-h light/dark cycle and free access to food and water. TGF mice were randomly divided into placebo (TGFp; $\mathrm{n}=7$ ) and PP supplementation (TGFpp; $\mathrm{n}=7$ ). PPs were administered in drinking water (50 $\mathrm{mg} / \mathrm{kg} / \mathrm{d}$ ) for $4 \mathrm{wk}$. The polyphenolic extract administered was commercialized by GRAP'SUD (Cruviers-Lascours, France) as Exgrape ${ }^{\circledR}$ (Lalilab Inc, Durham, NC, USA) total extract, whom biochemical characteristics have been previously published [34]. It contains more than 92\% of total PP as catechin equivalent, $15 \%$ procyanidines, $2 \%$ anthocyanes and $100 \mathrm{ppm}$ of resveratrol. All these polyphenolic compounds were extracted and purified from red grapes with processes that preserved their characteristics. Preceding dissection, animals were euthanized by rapid cervical dislocation.

\section{Morphometric analysis}

Tibialis anterior muscles (TAs) were dissected, immediately frozen in liquid nitrogen after being immersed in a solution of cold isopentane, and stored at $-80^{\circ} \mathrm{C}$ until further analysis. Ten $\mu \mathrm{m}$ TA transverse cryostat sections were stained with hematoxylin and eosin $(\mathrm{H} \& \mathrm{E})$. Stained sections were then viewed under a Nikon (Tokyo, Japan) optiphot-2 microscope and images were captured with the Microvision Instruments' (Cedex, France) camera driven by Histolab (Gothenburg, Sweden) program version 5-13-1 software (Microvision Instruments). Morphometric analysis was respectively performed on three different crosssections area (CSA) of each muscle studied using the Histolab program.

\section{Immunohistochemistry}

Ten $\mu \mathrm{m}$ TA cryostat sections were incubated with an anti-F4/80 antibody (AbDserotech, Kidlington, United Kingdom) for $1 \mathrm{~h}$ at room temperature. After being washed out with phosphate buffered saline solution, sections were incubated with an Alexa Fluor 488 conjugated antirat secondary antibody (Invitrogen) and DAPI. Fluorescence was viewed under a Leica Microscope (Leica DM6000, Wetzlar, Germany) using X63/1.40-0.60 HCX PL APO oil immersion objective. Images were captured as 16 TIFF files with the MicroMax 1300 CCD camera (RS-Princeton Instruments) driven by the MetaMorph (Nashville, TN, USA) (version 7; Universal Imaging, Bedford Hills, NY, USA) software.

\section{Mitochondrial isolation and respiration}

Mitochondria were isolated as previously described [35]. Rates of mitochondrial oxygen consumption were measured with a micro cathode oxygen electrode (Clark-type polarographic electrode) calibrated as previously described [35]. Fifty $\mu \mathrm{L}$ of isolated mitochondria were loaded into a respiratory chamber with respiration medium (RM) with pyruvate/malate as substrate and set at $37^{\circ} \mathrm{C}$ Adenosine 5-diphosphate (ADP) at a final concentration of $500 \mu \mathrm{M}$ was added to initiate state 3 respiration (ADP-stimulated). Respiratory control ratio (RCR) was obtained by dividing state 3 respiration rate by the recovery rate after ATP synthesis, i.e., state 4 respiration rate, defined as mitochondrial oxygen consumption after the depletion of exogenous ADP. ADP/O was expressed as number of nmol of ADP phosphorylated by nmol of $O$ consumed. Each measurement was made in triplicate. Oxygen consumption was specified as the amount of oxygen disappearing from the respiration chamber over time per milligram of mitochondrial protein ( $\mathrm{nmol} \mathrm{O/min/mg} \mathrm{protein).}$

\section{Total protein extract}

TA muscles were thoroughly blended using a hand held homogenizer (Kinematica AG - POLYTRON PT 1300 D, Luzern, Switzerland) in lysate buffer complemented with protease and phosphatase inhibitors (Sigma-Aldrich, St. Louis, MO, USA) and centrifuged $10 \mathrm{~min}$ at $1000 \mathrm{~g}$. Protein concentration of the supernatant was estimated with the BCA protein assay kit (Pierce).

\section{Immunoblotting}

Total or mitochondrial protein extracts were analyzed by western blot. Polyvinylidene fluoride (PVDF) membranes were incubated with the primary antibodies directed against: cytochrome C, 4-hydroxynonenal (4 HNE), I $\kappa \alpha$, caspase-9, caspase-3 (Santa Cruz Biotechnology, Dallas, TX, USA); antimyosin heavy chain (MHC) fast, anti- $\alpha$ tubulin (Millipore, Billerica, MA, USA); nitrotyrosine (Mitosciences); oxidative phosphorylation complexes proteins (OXPHOS; Amersham, England, UK); MnSOD, CuZn SOD (assay designs, Farmingdale, NY, USA); glutathione reductase (GR), and catalase (AbFrontier, Seoul, Korea). After primary antibody incubation, PVDF membranes were incubated with secondary antibodies conjugated to peroxidase (Sigma-Aldrich, St. Louis, MO, USA) and protein expression levels were quantified with Image software corrected with tubulin expression and normalized with CTL set at $100 \%$. Oxidized proteins were analyzed by western blot with the Oxyblot protein oxidation detection kit (Chemicon International, Temecula, CA, USA).

\section{Quantitative RT-PCR amplification}

Total RNAs were isolated from TA cells by blending muscle biopsies with a homogenizer (Kinematica AG - POLYTRON PT 1300 D) in the presence of the TRIzol reagent (Invitrogen, Omaha, NE, USA). To avoid genomic DNA contamination and amplification during PCR analysis, RNAs were treated with RNase-free DNase (Euromedex, Strasbourg, France) before their reverse transcription. Complementary DNAs (cDNAs) were generated by RT with oligo-(dT) primers and the Superscript II enzyme (Invitrogen). Gene sequences for primer design were 
Table 1

Primers sequences

\begin{tabular}{|c|c|c|}
\hline Genes & Sequences sens & Sequences antisens \\
\hline $\mathrm{TNF} \alpha$ & TACTGAACTTCGGGGTGATTGGTCC & CAGCCTTGTCCCTTGAAGAGAACC \\
\hline IFN $\gamma$ & GAACTGGCAAAAGGATGGTGACAT & CCGCTTCCTGAGGCTGGATT \\
\hline OAS1 & AGTCATAAACTACCAGCAACTCT & GGTCTCCACCACCCAAG \\
\hline OAS2 & CTGCTCACCATCTATGCCT & AGAAACTTCCTCACGGTCTC \\
\hline Cyto c & TCCATCAGGGTATCCTCTCC & GGAGGCAAGCATAAGACTGG \\
\hline PGC1 $\alpha$ & CCCGTGGATGAAGACGGATTG & GTGGGTGTGGTTTGCTGCATG \\
\hline TFAM & ATGTCTCCGGATCGTTTCAC & CСAAAAAGACCTCGTTCAGC \\
\hline MURF1 & GCAGCTCATCAAGAGCATTG & CAGAGCGTGTCTCACTCATC \\
\hline Atrogin-1 & GAGAATCTGTTCAGCAGCCT & ATCAGCTCCAACAGCCTTAC \\
\hline
\end{tabular}

TNF $\alpha$, tumor necrosis factor alpha; IFN $\gamma$, interferon $\gamma$; OAS, oligoadenylate synthetase; Cyto c, cytochrome c; PGC1 $\alpha$, peroxisome proliferator-activated receptor gamma coactivator 1 alpha; TFAM, mitochondrial transcription factor A; MURF1, muscle RING-finger protein-1.

obtained from the NCBI Reference Sequences database. Primer sequences are in Table 1. For PCR amplification ( 30 cycles) the amount of each cDNA was adjusted for each primer pair to be in the linear range of amplification and to give the same quantity of amplified complementary DNA as with the eukaryotic translation elongation factor $1 \alpha$ (eEF1 $\alpha$ ) primers. After $1.2 \%$ agarose gels analysis PCR products were quantified with Eastman Kodak 1 D3.6 software (Rochester, NY).

\section{Statistical analysis}

All quantitative data are reported as means value \pm SEM. One-way ANOVA and post hoc Bonferroni's multiple comparison tests were used to determine statistical significance between groups using SigmaStat 3.5 (San Jose, CA, USA) software. Bars not sharing a common letter are significantly different at $P<0.05$.

\section{Results}

Skeletal muscle of TGF mice develop inflammation and oxidative stress

TGF mice are characterized by high-grade inflammation $[33,36]$. However, as inflammation profile and oxidative stress

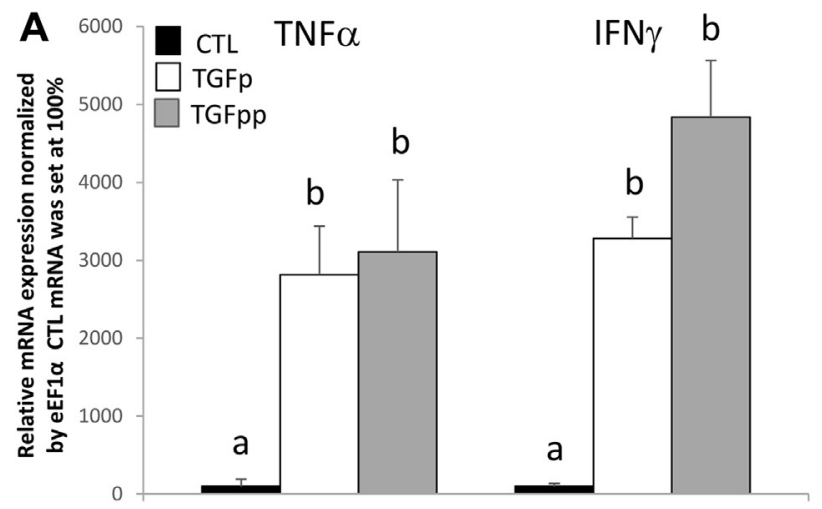

B

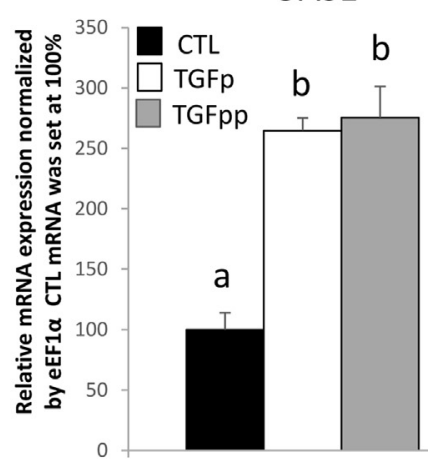

OAS2

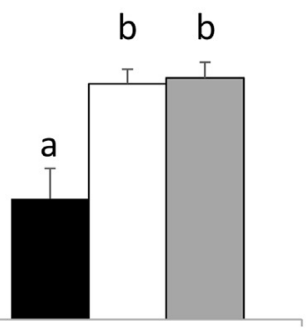

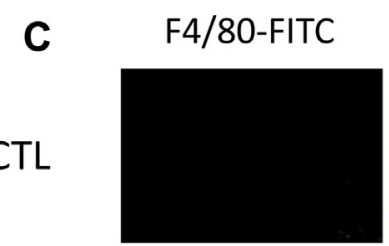

TGFp
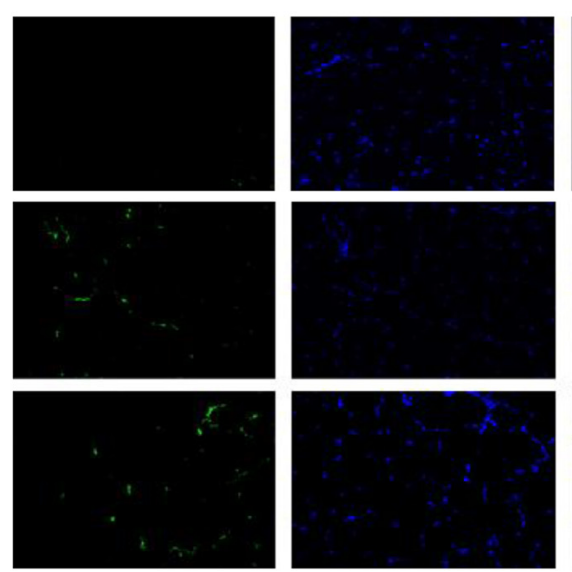
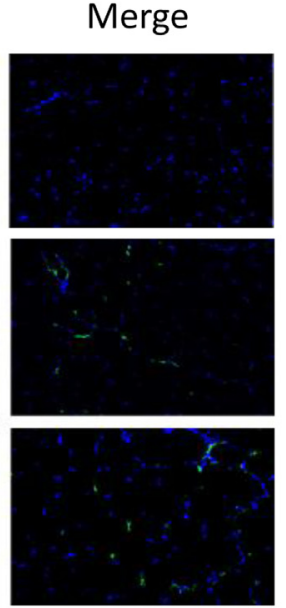

D

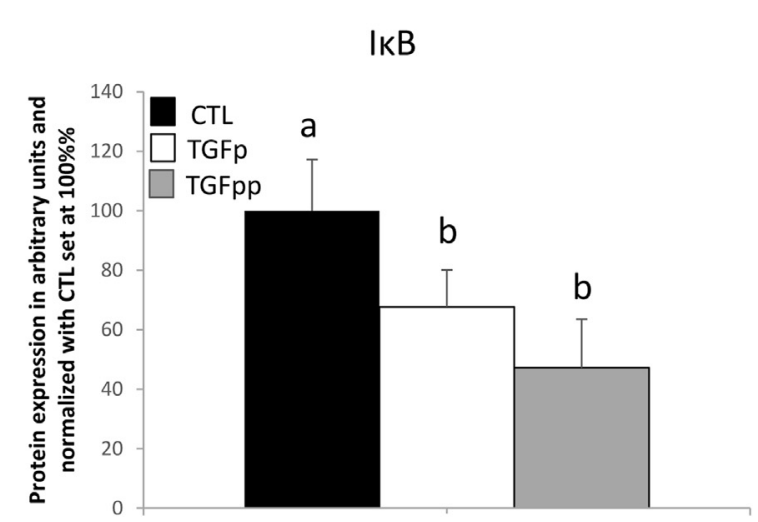

Fig. 1. TGF mice present skeletal muscle inflammation which is not prevented by polyphenol (PP) supplementation. (A, B) mRNA expression of TNF $\alpha$, IFN $\gamma$, and OAS1, OAS2 in SM of CTL (black), TGFp (white) and TGFpp (gray) mice. Data are expressed relative to the CTL value, which was set at $100 \%$. (C) Immunofluorescence analysis with anti-F4/80 antibody (green labeling) and nuclei labeling with DAPI (blue labeling) in SM. Merge illustrates macrophage infiltration with F4/80 and DAPI positive cells. (D) Quantification of I $\mathrm{B}$ protein expression corrected by $\alpha$ tubulin levels used as an indicator of proteins loading. Data are expressed relative to the CTL value, which was set at $100 \%$. eFN1a. All data are presented as average $\pm \mathrm{SEM} ; \mathrm{n} \geq 5$ by group. Bars not sharing a common letter are significantly different at $P<0.05$. 
have not been characterized in TGF mice skeletal muscle (TGFp SM), we first analyzed inflammation and oxidative stress in TGFp SM compared to control mice skeletal muscle (CTL SM).

Inflammatory cytokines TNF $\alpha$, IFN $\gamma$, and two interferon (IFN)stimulated genes oligoadenylate synthetase 1 and 2 (OAS1 and OAS2) mRNA levels were significantly increased in TGFp SM compared to CTL SM (Fig. 1A,B). Further, macrophage infiltration was increased in TGFp SM, whereas macrophages were only occasionally observed in CTL SM (Fig. 1C). In addition, as shown in Figure 1D, the amount of $\mathrm{I} \kappa \mathrm{B} \alpha$ (inhibitor of NF- $\kappa \mathrm{B}$ ) protein was significantly lower in TGFp SM than in CTL SM. These results were indicative of a high-grade inflammation state in TGFp SM.

TGFp SM was also characterized by a significant increase in nitrotyrosine proteins (Fig. 2A). 4 HNE-modified protein (4-hydroxy-2-nonenal) and carbonylated protein levels were also increased in TGFp SM, but without reaching significance (Fig. 2B,C). Moreover, no significant change in TGFp SM levels of antioxidative enzymes: super oxidative dismutases (CuZnSOD and MnSOD), catalase and glutathione reductase (GR) were observed (Fig. 2, panels D-G). These data suggest that oxidative stress could develop in TGFp SM consecutively to an overproduction of ROS, as there is no change in antioxidative enzymes levels.
Muscle mitochondria from TGF mice are characterized by increased oxidative stress and alteration of their respiratory chain function

Mitochondria are not only one of the major sources of ROS, but also the primary targets of oxidative-induced damage. Thus, we investigated oxidative stress and mitochondria activity on isolated mitochondria. Although there was no alteration in carbonylated mitochondrial protein level (Fig. 3A), the 4 HNE-modified protein level was significantly increased in isolated mitochondria of TGFp SM compared to CTL SM (Fig. 3B), suggesting mitochondria oxidative stress.

Isolated mitochondrial activity was first analyzed through oxygen consumption in the presence of pyruvate as substrate. Results are summarized in Table 2 . Basal state 2 respiration and state 4 , after ADP consumption, were not altered in mitochondria of TGFp SM compared to CTL SM. ADP-stimulated state 3 respiration was significantly decreased in mitochondria of TGFp SM compared to CTL SM. Consequently, the RCR was significantly decreased in mitochondria of TGFp SM.

In isolated mitochondria, there was no difference either in OXPHOS content (Fig. 3C) or in mitochondrial enzyme hydroxyacylCoA dehydrogenase (HADH) activity (Fig. 3D) between TGFp and CTL mice. Furthermore, expression of several
A
Nitrotyrosine
B
4HNE
C Carbonylated protein
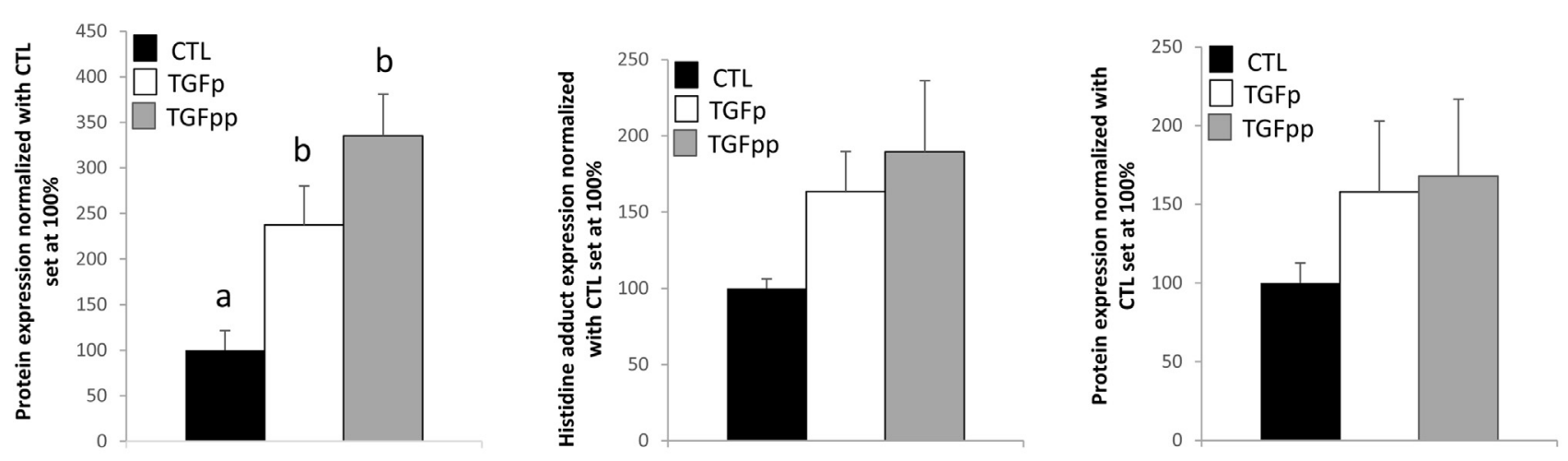

D

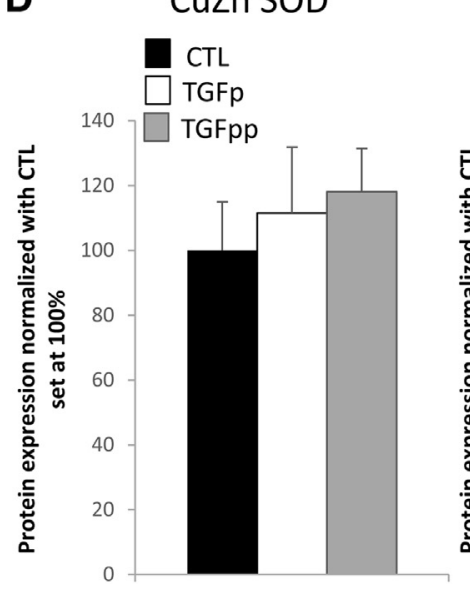

E $M n S O D$

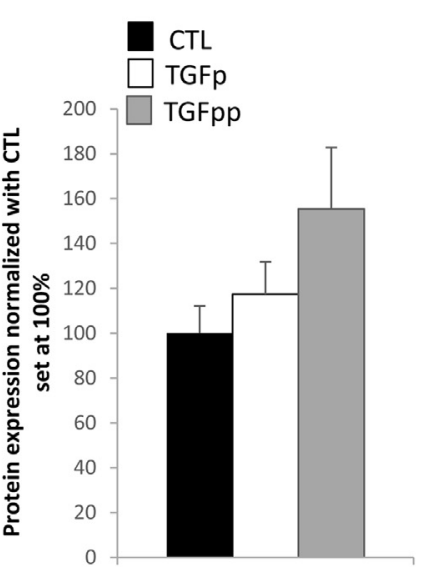

$\mathbf{F}$

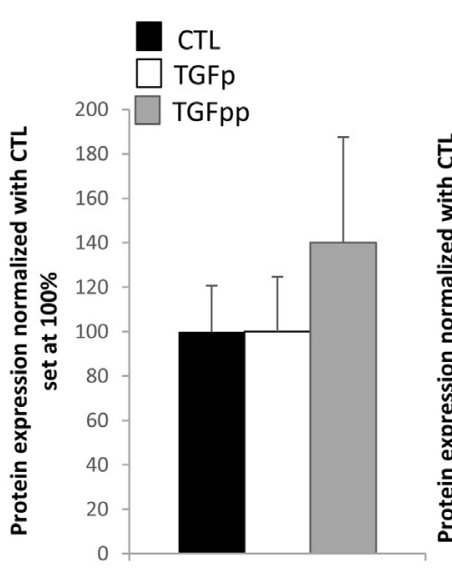

G

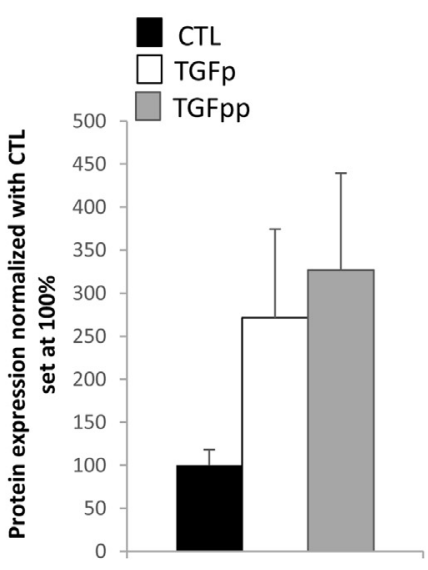

Fig. 2. Skeletal muscle of TGF mice present increase in oxidative stress markers not corrected by PP supplementation. Total protein extracts of TA of CTL (black), TGFp (white), and TGFpp (gray) mice were analyzed by western blot with antibodies directed against nitrotyrosine (A), 4HNE (B), carbonylated protein (C), CuZn (D), MnSOD (E), catalase (F) and glutathione reductase (GR) (G), protein expressions were corrected by $\alpha$ tubulin levels used as an indicator of proteins loading. All data are expressed relative to the CTL value, which was set at $100 \%$. All data are presented as average \pm SEM; $\mathrm{n} \geq 5$ mice by group. Bars not sharing a common letter are significantly different at $P<0.05$. 
A Carbonylated protein

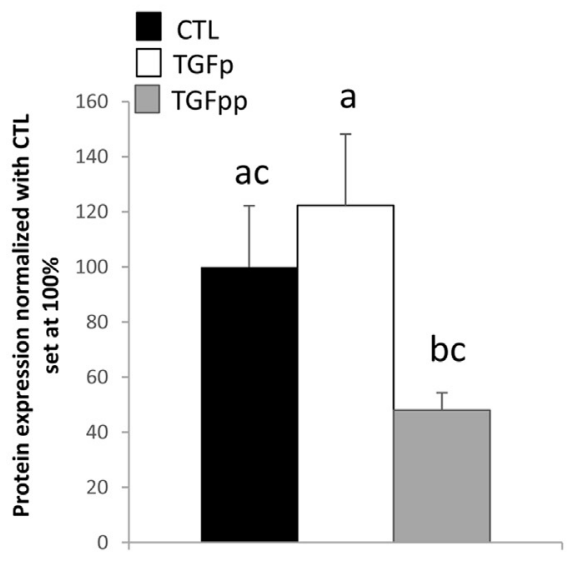

B

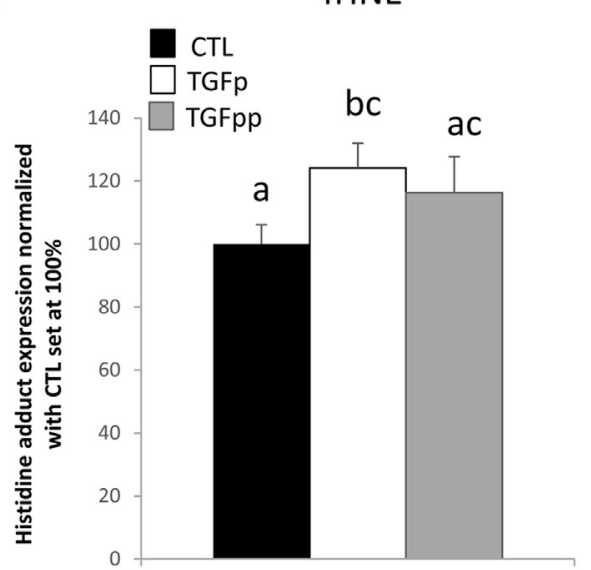

C

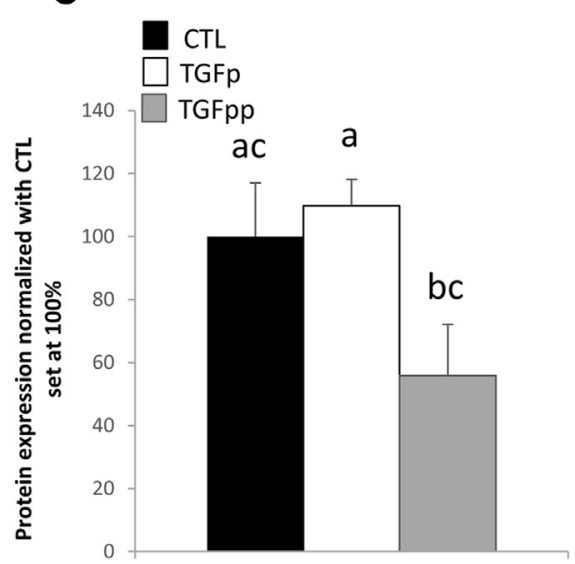

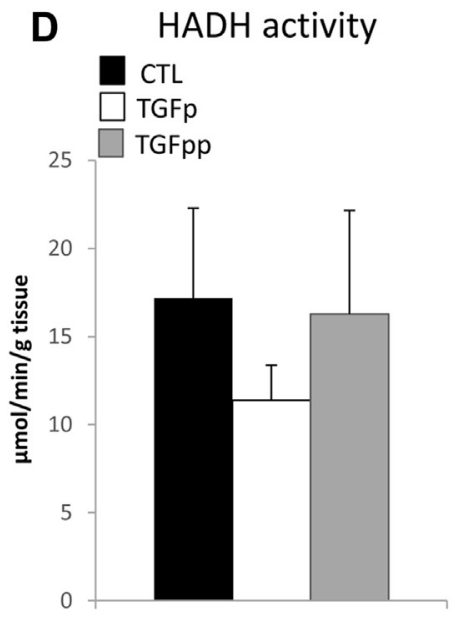

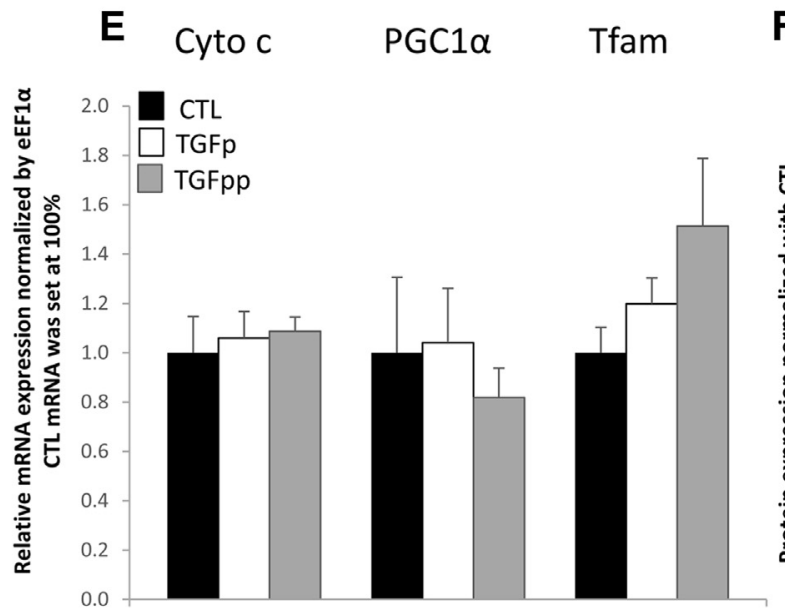

$\mathbf{F}$

Muscle OXPHOS

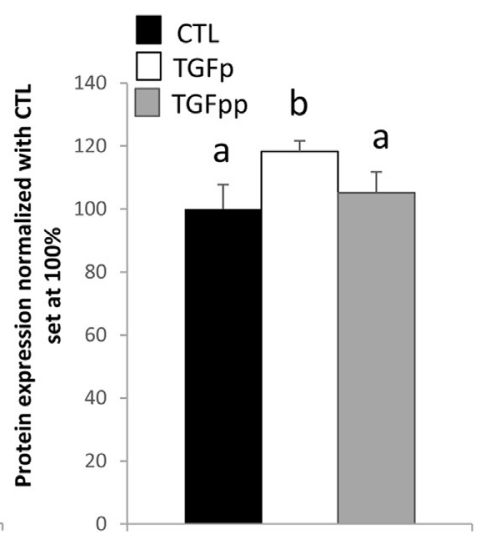

Fig. 3. Isolated mitochondria from skeletal muscle of TGF mice present alteration in oxidative stress marker partially reduce with polyphenol (PP) supplementation. Isolated mitochondria of CTL (black), TGFp (white) and TGFpp (gray) mice were analyzed by Western blot with antibodies directed against carbonylated proteins (A), 4HNE (B), and mitochondria OXPHOS (C). All data are expressed relative to the CTL value, which was set at $100 \%$. Mitochondrial activity was assessed with HADH activity (D). mRNA expression of mitochondria biogenesis genes: cytochrome c (cyto c), PGC1 $\alpha$, and Tfam (E). All data are expressed relative to the CTL value, which was set at $100 \%$. OXPHOS proteins were also investigated on whole muscle extract (F). Protein expressions were corrected by $\alpha$ tubulin levels used as an indicator of proteins loading. Data are expressed relative to the CTL value, which was set at $100 \%$. Data are presented as average \pm SEM; $n \geq 5$ mice by group. Bars not sharing a common letter are significantly different at $P<0.05$. Oxidative phosphorylation complexes proteins (OXPHOS).

genes were involved in mitochondrial biogenesis: Peroxisome proliferator-activated receptor gamma coactivator 1 alpha (PGC1 $\alpha$ ), mitochondrial transcription factor A (TFAM) and cytochrome $C$ were not significantly different between TGFp and CTL SM (Fig. 3E). Finally, when expressed per total amount of isolated mitochondrial OXPHOS protein content, the ADP/O ratio was not different in TGFp mice compared to CTL mice (Table 2). Interestingly, in muscle, OXPHOS protein content was significantly increased in TGFp SM extracts (Fig. 3F) suggesting an increase in mitochondria quantity. These results showed an increase in mitochondria oxidative damage associated with a higher OXPHOS content in TGFp SM extract with a decrease in mitochondrial respiration. These data suggest that SM mitochondria function was impaired in TGFp mice.

\section{TGF mice develop skeletal muscle atrophy}

SM weight (Fig. 4A) and mean values of SM fiber crosssectional areas (CSA) (Fig. 4, panels B-C) were significantly decreased in TGFp mice. Further, the left shift of fiber CSA frequency distribution curve (Fig. 4D) illustrated an increase in the percentage of smaller fibers and a decrease in the percentage of larger fibers, demonstrating atrophy of SM in TGFp mice. Moreover, mRNAs of ubiquitin ligases MuRF1 and Atrogin-1 were significantly increased in TGFp mice (Fig. 4E). In the same way, activation of caspases- 3 and 9 was increased in TGFp as attested by their higher level of cleaved fragment (Fig. 4F) and the myosin heavy chain (MHC) cleavage (Fig. 4, panels F-G). These data demonstrated that SM atrophy is associated with activation of caspases and UPS in TGFp SM.

\section{PP supplementation does not modulate skeletal muscle inflammation but reduces mitochondrial dysfunction}

To investigate the potential benefit of PP supplementation, TGF mice were supplemented with red grape polyphenol (TGFpp) or placebo (TGFp) for $4 \mathrm{wk}$. We analyzed TGFpp and TGFp SM for inflammation and oxidative stress. PP supplementation did not reduce inflammation in TGFpp SM, as attested by the absence of change in TNF $\alpha$, IFN $\gamma$, OAS1, and OAS2 mRNA levels (Fig. 1A,B), macrophage infiltration (Fig. 1C), and IkB level (Fig. 1D). Moreover, in SM extract, nitrotyrosine, 4 HNE-modified 
Table 2

Functional data obtained from skeletal muscle mitochondria isolated from control (CTL) and transgenic (TGF) mice supplemented (TGFpp) or not (TGFp) with polyphenols (PP)

\begin{tabular}{|c|c|c|c|}
\hline Mitochondrial respiration & CTL & TGFp & TGFpp \\
\hline State 2 (nmol $\mathrm{O} / \mathrm{min} / \mathrm{mg}$ protein) & $45 \pm 4$ & $39.2 \pm 3.6$ & $28.3 \pm 5.9$ \\
\hline State $3(\mathrm{nmol} \mathrm{O} / \mathrm{min} / \mathrm{mg}$ protein) & $387.8 \pm 37.7^{\mathrm{a}}$ & $277.6 \pm 30.9^{b}$ & $178.1 \pm 34^{c}$ \\
\hline State $4(\mathrm{nmol} \mathrm{O} / \mathrm{min} / \mathrm{mg}$ protein $)$ & $77 \pm 4.6$ & $72.2 \pm 4.8$ & $68.3 \pm 5.6$ \\
\hline $\mathrm{RCR}$ & $5.1 \pm 0.46^{\mathrm{a}}$ & $3.8 \pm 0.5^{\mathrm{b}}$ & $2.7 \pm 0.5^{c}$ \\
\hline ADP/O/ OXPHOS & $7.5 \times 10^{-3} \pm 1.2 \times 10^{-3 a}$ & $7.4 \times 10^{-3} \pm 0.8 \times 10^{-3 a}$ & $13.4 \times 10^{-3} \pm 2.5 \times 10^{-3 b}$ \\
\hline
\end{tabular}

RCR, respiratory control ratio; ADP, adenosine 5-diphosphate; OXPHOS, oxidative phosphorylation complexes proteins.

$\mathrm{ADP} / \mathrm{O}$ data are expressed as number of nmol of ADP phosphorylated by nmol of $\mathrm{O}$ consumed. All data are presented as the mean $\pm \mathrm{SEM}$; $\geq 5$ mice by group. Means not sharing a common letter are significantly different at $P<0.05$.

proteins and carbonylated protein levels were not decreased after PP supplementation (Fig. 2, panels A-C). Also, antioxidative enzymes (Fig. 2, panels D-G) and mitochondrial HADH activity (Fig. 3D) were not modified, nor was mitochondrial biogenesis genes expression (Fig. 3E).

We next investigated oxidative stress markers in isolated mitochondria. We did not observe a decrease in 4 HNE-modified protein levels (Fig. 3B). However, surprisingly, carbonylated protein content was significantly reduced after PP supplementation (Fig. 3A), suggesting a reduction in mitochondrial oxidative stress on some specific ROS. Regarding mitochondrial respiration, a significant decrease in state 3 was noticed, although basal and state 4 were not modified after PP supplementation (Table 2). Thus, TGFpp mitochondria presented a significant decrease in RCR compared to TGFp (Table 2). However, PP supplementation had no effect on the expression of OXPHOS proteins in SM extract (Fig. 3F), although it decreased the total amount of OXPHOS proteins in isolated mitochondria
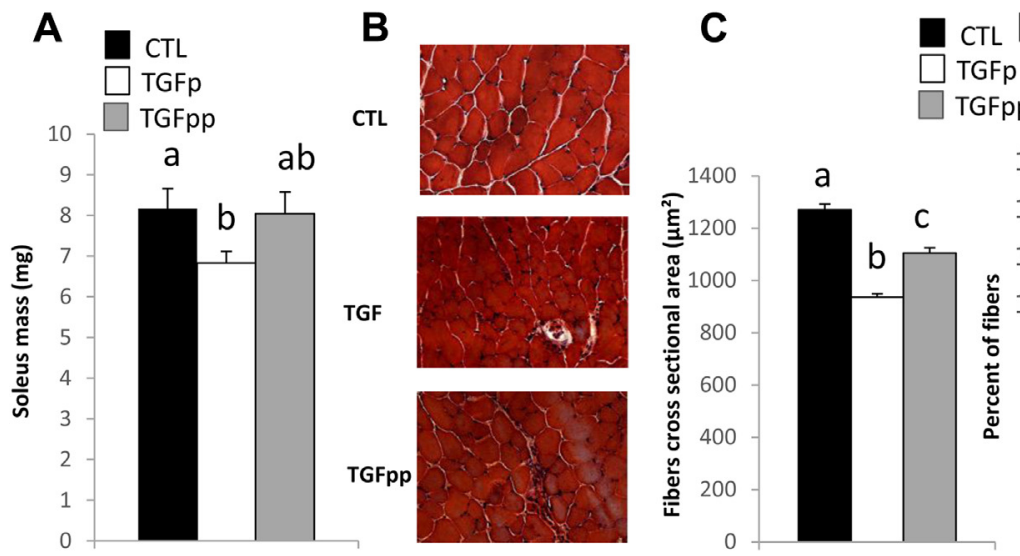

\section{Cross sectional area distribution} Fp Fpp
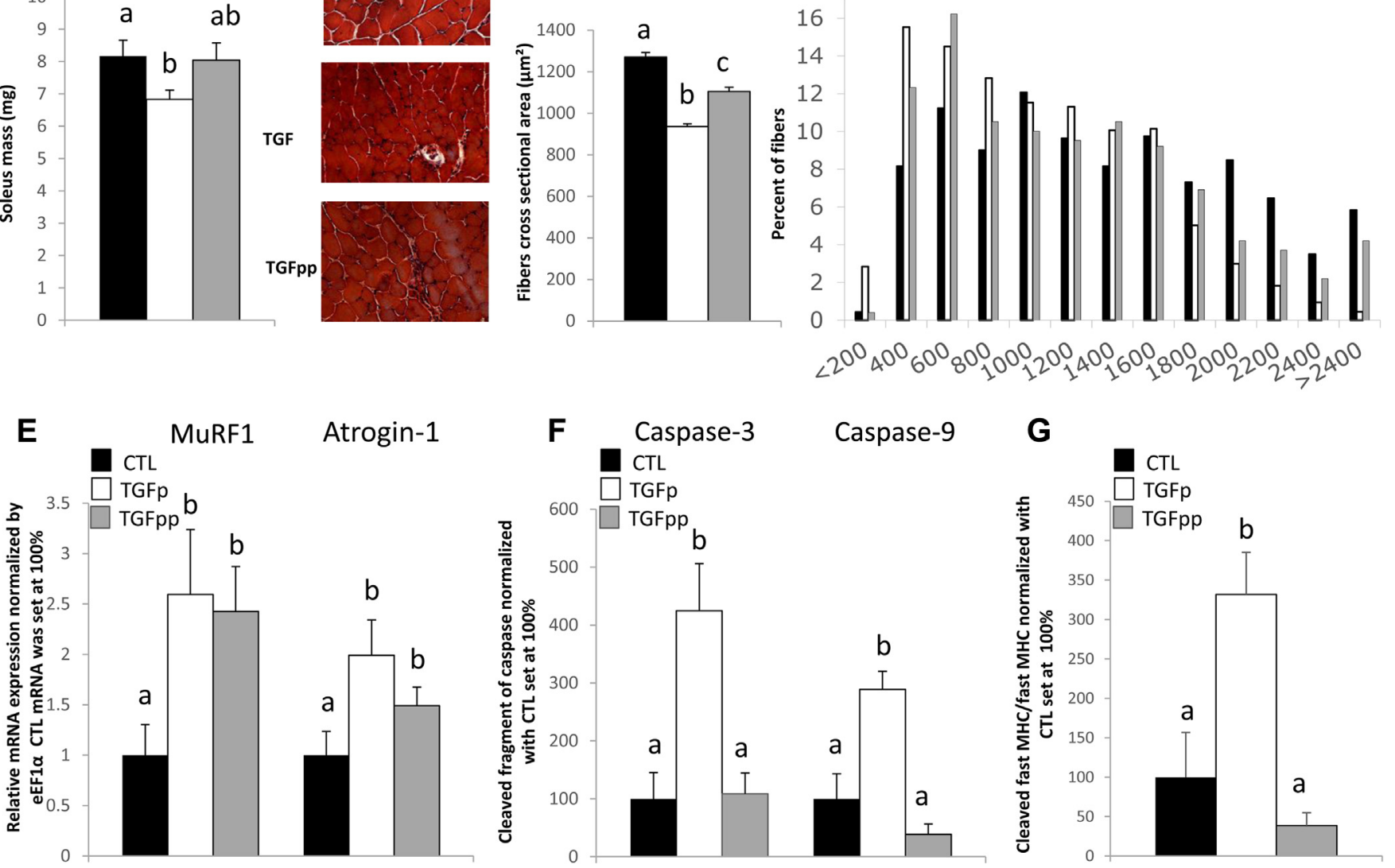

Fig. 4. TGF mice present skeletal muscle atrophy and increased atrophy gene expression and caspases-3/9 activation that mitigate with polyphenol (PP) supplementation. (A) Muscle weight of CTL (black), TGFp (white), and TGFpp (gray) mice. (B) Representative H \& E staining of transverse sections of frozen SM are shown at a 200 X magnification. (C) Muscle fiber cross-sectional area of $\sim 400$ fibers/muscle, from 3 mice/group. Data are presented as the average \pm SEM; $n \geq 1200$ by group. (D) Distribution of fiber crosssectional area. (E) mRNA expression of MuRF1 and Atrogin-1 in SM. All data are expressed relative to the CTL value, which was set at $100 \%$. (F) Cleaved fragment of caspase-3 and 9 in SM analyzed by Western blot corrected by $\alpha$ tubulin levels used as an indicator of proteins loading. All data are expressed relative to the CTL value, which was set at $100 \%$. (G) Protein level of cleaved fast MHC/fast MHC in SM. All data are presented as average \pm SEM; $n \geq 5$ by group. Bars not sharing a common letter are significantly different at $P<0.05$. Muscle RING-finger protein-1 (MuRF1); eukaryotic translation elongation factor $1 \alpha$ (eFA1 $\alpha$ ); myosin heavy chain (MHC). 


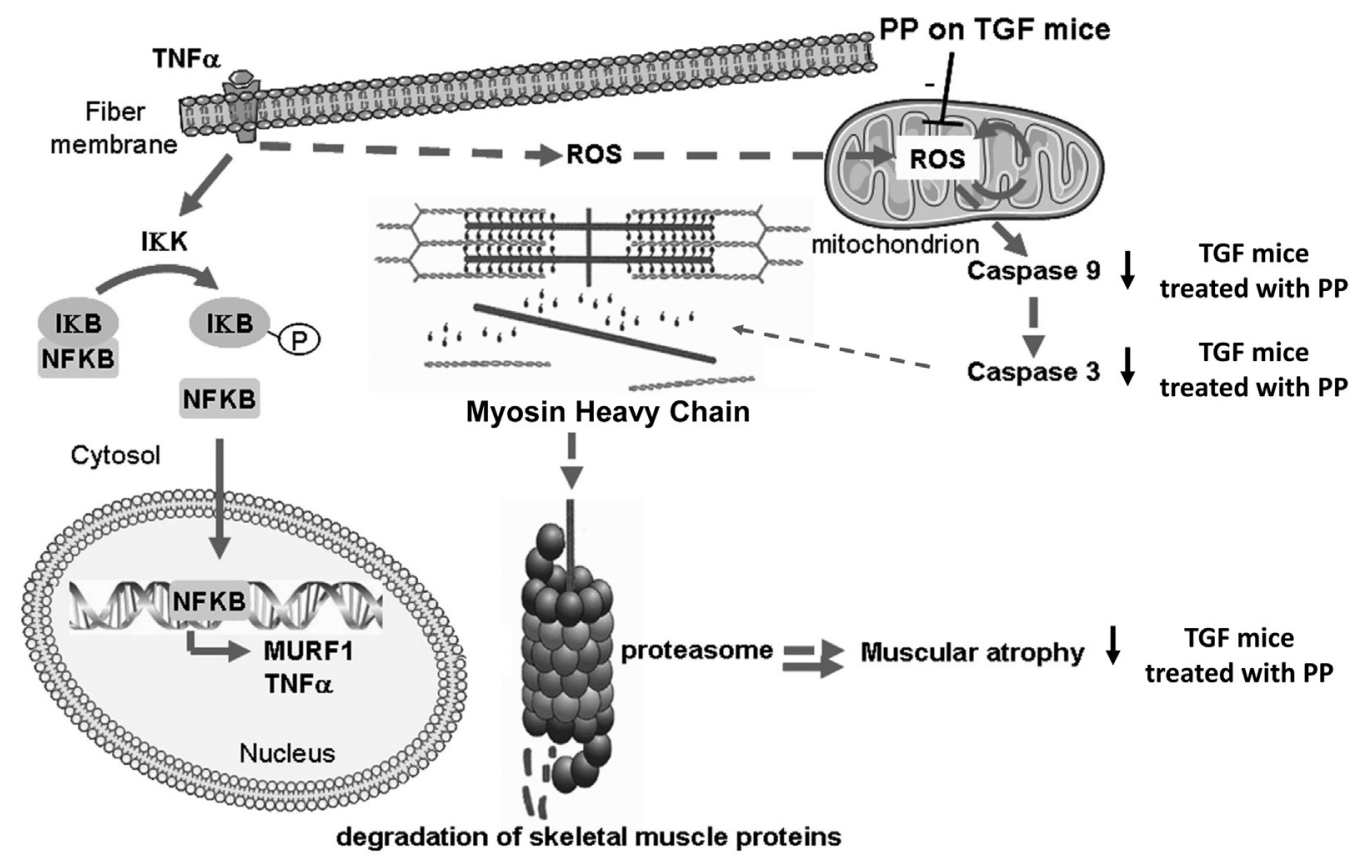

Fig. 5. Proposed overview of PP effects on two signaling pathways induced by TNF $\alpha$ and leading to muscular atrophy; in TGF mice PP act mainly on ROS signaling pathways with no effect on inflammation.

(Fig. 3C). Thus, mitochondrial efficiency measured with ADP/O was significantly increased after PP supplementation when normalized by the total amount of mitochondrial OXPHOS protein (Table 2) suggesting an improvement in mitochondrial function.

\section{PP supplementation reduces muscle atrophy in TGF mice}

Although increased muscle weight in TGFpp did not reach significance compared to TGFp (Fig. 4A), PP supplementation significantly increased mean CSA (Fig. 4, panels B-C) and a rightshift in fiber CSA frequency distribution curve was noticed (Fig. 4D). Thus, TGFpp SM had a decrease in smaller fibers percentage and an increase in larger fibers percentage. However, no change of the ubiquitin ligase MuRF1 and Atrogin-1 mRNAs levels was observed in TGFpp SM (Fig. 4E), while we observed a significant inhibition of activated caspases 3 and 9 expression (Fig. 4F). These results illustrated an inhibition of protein degradation pathways. As expected, TGFpp SM presented a significant reduction (88\%) in cleaved MHC level compared to TGFp SM (Fig. 4G). Overall, TGFpp SM presented no more significant difference compared to CTL SM except for MuRF1 and Atrogin-1 mRNA expression levels.

\section{Discussion}

We demonstrated to our knowledge for the first time, that TGF mice, characterized by T cell-driven inflammation, developed (i) SM inflammation and (ii) SM atrophy. We further demonstrated that red grape PP supplementation mitigates muscular atrophy in TGF mice, by notably acting on mitochondria function and caspase activation but not on Atrogin-1 and MuRF1 mRNAs levels.

TGF mice were previously characterized by a major systemic inflammation [32,33] with no data on muscle. Our results demonstrated that SM also developed an inflammatory state characterized by high expression of inflammatory gene (TNF $\alpha$,
IFN $\gamma$, and OAS1/OAS2) and NF- $\kappa$ B activation. Associated with this inflammation, TGF mice developed SM atrophy, described, to our knowledge for the first time, in this mice model.

We demonstrated that, despite an increased mitochondrial content in TGF SM, muscle atrophy was associated with mitochondrial dysfunction, increased mitochondrial oxidative stress, MuRF1 and Atrogin-1 genes expression and caspase- 3 and 9 activation. Based on previous studies $[11,13,14]$, we speculate that TGF SM mitochondria produced excessive amount of ROS, resulting in a deleterious effect on mitochondrial proteins leading to mitochondrial dysfunction. As previously described [37], our data highlight the role of mitochondria in the development of muscle atrophy. In fact, both disruption/impairment of mitochondrial function, network or mitophagy lead to the accumulation of damaged and dysfunctional mitochondria [38,39] that contribute to muscle atrophy $[7,40]$.

We found that nutritional amount of grape PP was sufficient to mitigate muscle atrophy. As chronic inflammation in TGF mice cannot be negatively regulated [32], PP supplementation did not reduce muscular inflammation. Our data showed that PP supplementation in TGF mice acted mainly on ROS damage. PP supplementation reduced oxidized mitochondrial proteins, improved mitochondrial function and dramatically reduced caspases- 9 and 3 activation. The TNF $\alpha / N F-\kappa B / M u R F 1-A t r o g i n-1 /$ atrophy pathway was not affected by PP supplementation. Neither NF- $\kappa$ B activation nor the increased expression of TNF $\alpha$ and MuRF1-Atrogin-1 mRNA were decreased after PP supplementation. Although mRNA levels do not reflect exactly protein levels and activity, we speculate that MuRF1 and Atrogin-1 activity are increased in TGF mice secondary to their inflammatory status since these genes are known to be mainly regulated by inflammation [41]. Finally, this pathway appears to be independent of ROS regulation. In contrast, the caspase- 9 and 3 activation were partially reverted in TGFpp (Fig. 5) indicating that their activation is dependent on ROS production. PP supplementation was not able to decrease the ROS-mediated formation of reactive aldehydes (i.e., $4 \mathrm{HNE}$ ) in both $\mathrm{SM}$ extract as in 
isolated mitochondria, whereas there was a reduction in oxidized mitochondrial protein and decreased caspase- 3 and 9 activation. These data suggest that PP supplementation did not have an impact on all sources of ROS.

Beneficial effects of PP supplementation on muscle atrophy appeared to be mediated through modulation of mitochondria function and caspase activation, as previously found in lowgrade inflammation or muscle inactivity models [42,43].

\section{Conclusion}

Our results demonstrated to our knowledge for the first time, that in a murine model of chronic high-grade inflammation (TGF mice), characterized by SM inflammation, oxidative stress, mitochondrial dysfunction and muscle atrophy, a nutritional amount of red grape PP supplementation mitigates muscle atrophy, by principally acting at mitochondrial level. In fact, PP supplementation improves mitochondrial function and decreases caspases activation, even without acting on Atrogin-1 and MuRF1 expression.

Thus, supplementation with a nutritional amount of red grape PP might be beneficial for reducing SM atrophy and possibly improving quality of life and life expectancy in chronic high-grade inflammatory diseases. This beneficial effect needs to be validated in further human studies.

\section{Acknowledgments}

Mouse model was generously donated by Pr Göran Hansson, Experimental Cardiovascular Medicine, Karolinska Hospital, Stockholm, Sweden. The authors thank Marie Hokayem (UMR CNRS 9214 - Inserm U1046) for language editing.

This work was supported by grants from INSERM and "Société Francophone du Diabète" (SFD) to A.S.; M.C.-Q. was supported by the "Société de Physiologie" (SP).

\section{References}

[1] Callahan LA, Supinski GS. Sepsis-induced myopathy. Crit Care Med 2009;37:S354-67.

[2] Mastrocola R, Reffo P, Penna F, Tomasinelli CE, Boccuzzi G, Baccino FM, et al. Muscle wasting in diabetic and in tumor-bearing rats: role of oxidative stress. Free Radic Biol Med 2008;44:584-93.

[3] Marquis K, Debigare R, Lacasse Y, LeBlanc P, Jobin J, Carrier G, et al. Midthigh muscle cross-sectional area is a better predictor of mortality than body mass index in patients with chronic obstructive pulmonary disease. Am J Respir Crit Care Med 2002;166:809-13.

[4] Desmeules S, Levesque R, Jaussent I, Leray-Moragues H, Chalabi L, Canaud B. Creatinine index and lean body mass are excellent predictors of long-term survival in haemodiafiltration patients. Nephrol Dial Transplant 2004;19:1182-9.

[5] Bodine SC, Latres E, Baumhueter S, Lai VK, Nunez L, Clarke BA, et al Identification of ubiquitin ligases required for skeletal muscle atrophy. Science 2001;294:1704-8.

[6] Sandri M, Sandri C, Gilbert A, Skurk C, Calabria E, Picard A, et al. FOXO transcription factors induce the atrophy-related ubiquitin ligase atrogin-1 and cause skeletal muscle atrophy. Cell 2004;117:399-412.

[7] Sandri M. Protein breakdown in muscle wasting: role of autophagylysosome and ubiquitin-proteasome. Int $\mathrm{J}$ Biochem Cell Biol 2013;45:2121-9.

[8] Sanchez AM, Bernardi H, Py G, Candau R. Autophagy is essential to support skeletal muscle plasticity in response to endurance exercise. Am J Physiol Regul Integr Comp Physiol 2014;307:956-69.

[9] Supinski GS, Wang W, Callahan LA. Caspase and calpain activation both contribute to sepsis-induced diaphragmatic weakness. J Appl Phys 2009;107:1389-96.

[10] Reid MB, Li YP. Cytokines and oxidative signaling in skeletal muscle. Acta Physiol Scand 2001;171:225-32.

[11] Reid MB, Moylan JS. Beyond atrophy: redox mechanisms of muscle dysfunction in chronic inflammatory disease. J Physiol 2011;589:2171-9.
[12] Gomes-Marcondes MC, Tisdale MJ. Induction of protein catabolism and the ubiquitin-proteasome pathway by mild oxidative stress. Cancer Lett 2002;180:69-74.

[13] Powers SK, Kavazis AN, McClung JM. Oxidative stress and disuse muscle atrophy. J Appl Phys 2007;102:2389-97.

[14] Powers SK, Kavazis AN, DeRuisseau KC. Mechanisms of disuse muscle atrophy: role of oxidative stress. Am J Physiol Regul Integr Comp Physiol 2005;288:337-44.

[15] Du J, Wang X, Miereles C, Bailey JL, Debigare R, Zheng B, et al. Activation of caspase-3 is an initial step triggering accelerated muscle proteolysis in catabolic conditions. J Clin Invest 2004;113:115-23.

[16] McClung JM, Kavazis AN, DeRuisseau KC, Falk DJ, Deering MA, Lee Y, et al. Caspase-3 regulation of diaphragm myonuclear domain during mechanical ventilation-induced atrophy. Am J Respir Crit Care Med 2007;175:150-9.

[17] Beyer I, Mets T, Bautmans I. Chronic low-grade inflammation and agerelated sarcopenia. Curr Opin Clin Nutr Metab Care 2012;15:12-22.

[18] Decendit A, Mamani-Matsuda M, Aumont V, Waffo-Teguo P, Moynet D, Boniface K, et al. Malvidin-3-O-beta glucoside, major grape anthocyanin, inhibits human macrophage-derived inflammatory mediators and decreases clinical scores in arthritic rats. Biochem Pharmacol 2013;86:1461-7.

[19] Mossalayi MD, Rambert J, Renouf E, Micouleau M, Merillon JM. Grape polyphenols and propolis mixture inhibits inflammatory mediator release from human leukocytes and reduces clinical scores in experimental arthritis. Phytomedicine 2014;21:290-7.

[20] Riegsecker S, Wiczynski D, Kaplan MJ, Ahmed S. Potential benefits of green tea polyphenol EGCG in the prevention and treatment of vascular inflammation in rheumatoid arthritis. Life Sci 2013;93:307-12.

[21] Avignon A, Hokayem M, Bisbal C, Lambert K. Dietary antioxidants: do they have a role to play in the ongoing fight against abnormal glucose metabolism? Nutrition 2012;28:715-21.

[22] Rahman I, Biswas SK, Kirkham PA. Regulation of inflammation and redox signaling by dietary polyphenols. Biochem Pharmacol 2006;72:1439-52.

[23] Wallerath T, Deckert G, Ternes T, Anderson H, Li H, Witte K, et al. Resveratrol, a polyphenolic phytoalexin present in red wine, enhances expression and activity of endothelial nitric oxide synthase. Circulation 2002; 106:1652-8.

[24] Holmes-McNary M, Baldwin AS Jr. Chemopreventive properties of transresveratrol are associated with inhibition of activation of the IkappaB kinase. Cancer Res 2000;60:3477-83.

[25] Yang EJ, Lee J, Lee SY, Kim EK, Moon YM, Jung YO, et al. EGCG attenuates autoimmune arthritis by inhibition of STAT3 and HIF-1 alpha with Th17| Treg control. PLoS One 2014;9:e86062.

[26] Hokayem M, Blond E, Vidal H, Lambert K, Meugnier E, Feillet-Coudray C, et al. Grape polyphenols prevent fructose-induced oxidative stress and insulin resistance in first-degree relatives of type 2 diabetic patients. Diabetes Care 2013;36:1454-61.

[27] Timmers S, Konings E, Bilet L, Houtkooper RH, van de Weijer T, Goossens GH, et al. Calorie restriction-like effects of 30 days of resveratrol supplementation on energy metabolism and metabolic profile in obese humans. Cell Metab 2011;14:612-22.

[28] Brasnyo P, Molnar GA, Mohas M, Marko L, Laczy B, Cseh J, et al. Resveratrol improves insulin sensitivity, reduces oxidative stress and activates the Akt pathway in type 2 diabetic patients. Br J Nutr 2011;106:383-9.

[29] Tangney CC, Rasmussen HE. Polyphenols, inflammation, and cardiovascular disease. Curr Atheroscler Rep 2013;15:324.

[30] Wang B, Yang G, Liang X, Zhu M, Du M. Grape seed extract prevents skeletal muscle wasting in interleukin 10 knockout mice. BMC Complement Altern Med 2014;14:162.

[31] Busquets S, Fuster G, Ametller E, Olivan M, Figueras M, Costelli P, et al. Resveratrol does not ameliorate muscle wasting in different types of cancer cachexia models. Clin Nutr 2007;26:239-44.

[32] Gorelik L, Flavell RA. Abrogation of TGF-beta signaling in T cells leads to spontaneous $\mathrm{T}$ cell differentiation and autoimmune disease. Immunity $2000 ; 12: 171-81$.

[33] Oertelt S, Lian ZX, Cheng CM, Chuang YH, Padgett KA, He XS, et al. Antimitochondrial antibodies and primary biliary cirrhosis in TGF-beta receptor II dominant-negative mice. J Immunol 2006;177:1655-60.

[34] Auger C, Gerain P, Laurent-Bichon F, Portet K, Bornet A, Caporiccio B, et al. Phenolics from commercialized grape extracts prevent early atherosclerotic lesions in hamsters by mechanisms other than antioxidant effect. J Agric Food Chem 2004;52:5297-302.

[35] Lambert K, Py G, Robert E, Mercier J. Does high-sucrose diet alter skeletal muscle and liver mitochondrial respiration? Horm Metab Res 2003;35:546-50.

[36] Yang GX, Lian ZX, Chuang YH, Moritoki Y, Lan RY, Wakabayashi K, et al. Adoptive transfer of CD8( + ) T cells from transforming growth factor beta receptor type II (dominant negative form) induces autoimmune cholangitis in mice. Hepatology 2008;47:1974-82.

[37] Romanello V, Guadagnin E, Gomes L, Roder I, Sandri C, Petersen Y, et al. Mitochondrial fission and remodelling contributes to muscle atrophy Embo J 2010;19:1774-85. 
[38] Lee HC, Yin PH, Chi CW, Wei YH. Increase in mitochondrial mass in human fibroblasts under oxidative stress and during replicative cell senescence. J Biomed Sci 2002;9:517-26.

[39] Jang YC, Lustgarten MS, Liu Y, Muller FL, Bhattacharya A, Liang $\mathrm{H}$, et al. Increased superoxide in vivo accelerates age-associated muscle atrophy through mitochondrial dysfunction and neuromuscular junction degeneration. FASEB J 2010;24:1376-90.

[40] Grumati P, Coletto L, Sabatelli P, Cescon M, Angelin A, Bertaggia E, et al. Autophagy is defective in collagen VI muscular dystrophies, and its reactivation rescues myofiber degeneration. Nat Med 2010;16:1313-20.
[41] Li YP, Chen Y, John J, Moylan J, Jin B, Mann DL, et al. TNF-alpha acts via p38 MAPK to stimulate expression of the ubiquitin ligase atrogin1/MAFbx in skeletal muscle. FASEB J 2005;19:362-70.

[42] Bennett BT, Mohamed JS, Alway SE. Effects of resveratrol on the recovery of muscle mass following disuse in the plantaris muscle of aged rats. PLoS One 2013;8:e83518.

[43] Momken I, Stevens L, Bergouignan A, Desplanches D, Rudwill F, Chery I, et al. Resveratrol prevents the wasting disorders of mechanical unloading by acting as a physical exercise mimetic in the rat. FASEB J 2011;25:3646-60. 